\title{
Defining the content of a minimal dataset for acquired brain injury using a Delphi procedure
}

\author{
Anne-Fleur Domensino ${ }^{1,2}$, leke Winkens ${ }^{2,3}$, Jolanda C. M. van Haastregt ${ }^{4}$, Coen A. M. van Bennekom ${ }^{5,6}$ and
} Caroline M. van Heugten ${ }^{1,2,3^{*}}$

\begin{abstract}
Background: The lack of consistency in outcome measurement within the field of acquired brain injury (ABI) leads to incomparability of collected data and, consequently, reduced generalisation of findings. We aim to develop a set of standardised measures which can be used to obtain the minimum amount of data necessary to characterise ABIpatients across all healthcare sectors and disciplines and in every stage of recovery; i.e., an ABI-specific minimal dataset (MDS-ABI). The current study was conducted to identify the core outcome domains for adults with ABI (what to measure?) and to select the most suitable measurements within these domains (how to measure it?).

Methods: An initial comprehensive set of outcome domains and measurement instruments relevant for measuring the consequences of $\mathrm{ABI}$ was identified by a literature study. The selection of relevant domains was based on the International Classification of Functioning, Disability and Health framework. Measurement instruments were included in the Delphi procedure when they met pre-set requirements. A three-round Delphi study was conducted among Dutch experts $(n=48)$ using iterative web-based surveys to prioritise the proposed domains and instruments for the MDS-ABI. Throughout all rounds, participants could recommend additional or alternative domains and measurement instruments, and were fed back the collated group responses of the previous round.

Results: Response rates ranged from 89 to $100 \%$. After three rounds, the expert panel reached consensus ( $\geq 51 \%$ ) on the inclusion of 12 outcome domains (demographics, injury characteristics, comorbidity, cognitive functioning, emotional functioning, energy, mobility, self-care, communication, participation, social support and quality of life), measured with six measurement instruments, two screening questions and a registry of demographic- and injury information. No consensus was reached on how to measure quality of life.
\end{abstract}

Conclusions: The current study achieved consensus on the content of a minimal dataset for patients with ABI. The current version of the MDS-ABI will be evaluated and optimised if necessary in the near future.

Keywords: Acquired brain injury, Minimal dataset, FAIR data, Delphi procedure, International Classification of Functioning, Disability and Health

\footnotetext{
* Correspondence: caroline.vanheugten@maastrichtuniversity.nl

${ }^{1}$ School for Mental Health and Neuroscience, Faculty of Health, Medicine and

Life Sciences, Maastricht University Medical Center, Maastricht, The

Netherlands

${ }^{2}$ Limburg Brain Injury Center, Maastricht, The Netherlands

Full list of author information is available at the end of the article
}

(c) The Author(s). 2020 Open Access This article is distributed under the terms of the Creative Commons Attribution 4.0 International License (http://creativecommons.org/licenses/by/4.0/), which permits unrestricted use, distribution, and reproduction in any medium, provided you give appropriate credit to the original author(s) and the source, provide a link to the Creative Commons license, and indicate if changes were made. The Creative Commons Public Domain Dedication waiver (http://creativecommons.org/publicdomain/zero/1.0/) applies to the data made available in this article, unless otherwise stated. 


\section{Background}

Acquired brain injury (ABI) encompasses all types of damage to the brain that occur after birth and are not related to progressive diseases. As such, ABI typically results in a sudden decrease of functioning in various domains. Research into the sequelae of ABI in all of these domains leads to an abundance of data. These data, however, lack uniformity due to the broad spectrum of available measurement instruments, leading to incomparability of collected data and, consequently, reduced generalisation of findings [1]. Moreover, suboptimal coordination of instrument use could lead to unnecessary or duplicate data administration when patients receive care in different healthcare sectors or within multiple healthcare disciplines, leading to the need for patients to 'repeat their story'. At the same time, the standardisation of outcome measurement could improve interdisciplinary communication and collaboration within healthcare for patients with ABI.

Recently, several studies have been conducted into the use of measurement tools in healthcare and research within the ABI population. For example, Tate et al. [2] systematically reviewed assessment tools used with adults in traumatic brain injury (TBI) research, identifying 728 unique instruments in several domains. Also, several systematic reviews into the measures of a particular domain have been conducted. For instance, Polinder et al. [3] identified 36 health-related quality of life measures used in TBI research, and recommended the establishment of consensus on methods of preference to facilitate comparability across studies. Furthermore, Tse et al. [4] reviewed participation measures used with stroke survivors and found 18 different measures, with none covering all aspects of participation as proposed by the International Classification of Functioning, Disability and Health (ICF) [5]. In conclusion, all studies revealed a high degree of variability in the use of measurement instruments, highlighting the need for consensus on preferred instruments for measuring outcome after ABI.

From a more general perspective, recent developments in the field of data collection promote findable, accessible, interoperable and reusable (FAIR) data, emphasizing the need for comparable data that can be integrated from non-cooperating resources with minimal effort [6]. Furthermore, international researchers, policymakers and clinicians have recognised the significance of FAIR data in healthcare by developing standardised outcome sets for specific populations or stages of a condition other than ABI [7-9].

Likewise, some initiatives have been undertaken for streamlining data collection in the field of ABI. One example is the National Institute of Health (NIH) recommendation to identify and use Common Data Elements with research in the field of neuroscience, such as the core dataset that was developed by Hicks et al. [10], aiding the standardised classification of injury severity after TBI. However, no Common Data Elements have been proposed for use with research into $\mathrm{ABI}$ in general. Similarly, the NIH recommends the use of PROMIS, a set of standardised generic patient report outcome measures $[10,11]$, comprehensive health-related quality of life measures specific for patients with neurologic conditions (NEURO-QOL) [12], and extensive standardised test batteries in several cognitive and emotional domains (NIH Toolbox) [13]. Another attempt at standardising data collection has been conducted by the International Consortium for Health Outcomes Measurement (ICHOM). The ICHOM initiative composes working groups of experts on specific health conditions to configure outcome sets that reflect what is most important to patients [14]. To date, one ICHOM set for stroke has been developed, but has yet to be evaluated [15]. Furthermore, the ICHOM sets tend to focus more on the neurological factors related to stroke. Hence, to date, there is no validated, compact dataset available for use in health care and research into all types and stages of ABI.

In the current project, we aim to develop a minimal dataset for adults with ABI (MDS-ABI). A minimal dataset is defined as a set of standardised measures used to index the minimum amount of data that is crucial for obtaining a global image of the patient across all healthcare sectors and disciplines and in every stage of the injury. It does not imply that outcome measurement needs to be restricted to the items that are in the set, as it can be expanded with measures of choice that serve a specific (research) goal [16]. The MDS-ABI aims to facilitate FAIR data collection on persons with $\mathrm{ABI}$ in the context of care, evaluation and research to improve the comparability of data, interdisciplinary communication and collaboration within the field of $\mathrm{ABI}$, while reducing the burden of questionnaire administration for patients and healthcare professionals. This study was conducted to identify the core outcome domains for adults with ABI (what to measure?) and to select the most suitable measurements within these domains (how to measure it?).

\section{Methods \\ Design}

The current study comprised an online three-round Delphi procedure $[17,18]$ among experts on measurement instruments used in the field of ABI.

\section{Participants}

Initially, potential experts were identified based on the professional network of the authors (criterion sampling). Invitees were then requested to propose supplementary 
potential experts within their own network (snowball sampling). Inclusion criteria were: being experienced in developing and/or evaluating measurement instruments that can be used to assess adults with $\mathrm{ABI}$ and being or having been employed in rehabilitation, neurology/ neurosurgery, neuropsychiatry, elderly care, or disability care in the Netherlands.

\section{Procedure}

Potential experts were asked by email to participate, before being admitted to the actual procedure. A briefing letter concerning the project, providing the outline and exact planning of the procedure was included with the e-mail. Potential participants were asked to judge whether they met the inclusion criteria and were willing to participate. Upon enrolment, participants provided information on their age, gender, occupation and experience with developing/evaluating measurement instruments. Participants received no compensation.

\section{Preparation phase}

In the preparation phase, a literature study into outcome domains and measurement instruments relevant to measuring the consequences of $\mathrm{ABI}$ was conducted. First, identification of potentially relevant domains was done by the authors prior to consulting the experts and was based on the ICF model [5] and the ICF core sets for stroke [19] and traumatic brain injury [20]. Because we anticipated that some domains (such as mental functions) are more applicable to ABI than others, these domains were further divided into second-level categories (such as emotional functioning). Furthermore, since health-related quality of life is an important outcome measure in $\mathrm{ABI}$ research, but is not yet a confirmed domain in the ICF model [21], it was decided that this domain be proposed as a separate factor. Last, the ICF categories 'activities' and 'participation' were merged, since they have proven to be difficult to differentiate in outcome measurement [22]. A discussion among members of an advisory group consisting of persons with $\mathrm{ABI}$ and their informal caregivers $(n=17)$ confirmed the relevance of the domains that were identified by the researchers.

Second, we looked into measurement use in the field of ABI. In order to improve compatibility with other data collection initiatives and current clinical practice, the authors determined that the minimal dataset should be composed of existing measurement instruments. Accordingly, we made an inventory of measurement use in large studies and other data-shaping initiatives [15, 23-25] and benchmarks [26] in the field of ABI. Furthermore, the systematic review by Tate [2] and her compendium of tools used for measuring the outcomes of ABI [27] were consulted and served as a guide for classifying measurement instruments within ICF categories. Finally, evidence-based guidelines of several healthcare disciplines and sectors were checked for recommendations regarding the use of measurement instruments.

A list of requirements for analysing the suitability of measurement instruments for the MDS-ABI was composed through a survey study among members of the knowledge network that the current project is part of $(n=11)$. This network is comprised of Dutch healthcare professionals and researchers aiming to increase knowledge, cooperation and communication within the field of ABI. The survey study involved a digital form containing proposed requirements for measurement instruments in the MDS-ABI. Respondents reviewed the suggested requirements (necessary/preferable/unnecessary), leading to a set of requirements and preferences, as displayed in Table 1.

All measurement instruments that were identified in the literature study were checked with our criteria and, when meeting all requirements, were entered into the first Delphi round.

\section{Delphi rounds}

The executive phase of the study comprised a threeround Delphi procedure. Experts who agreed to participate received a personal invitation by e-mail containing an anonymised web link to the first Delphi round. In this round, participants were asked to indicate whether every

Table 1 Requirements and preferences to guide the inclusion of existing measurement instruments

\begin{tabular}{|c|c|c|}
\hline Availability & Psychometric properties & Usability/feasibility \\
\hline \multicolumn{3}{|l|}{ Requirements } \\
\hline $\begin{array}{l}\text { - The instrument must be available in Dutch. } \\
\text { - The instrument must be in the public domain } \\
\text { and thus freely available. }\end{array}$ & $\begin{array}{l}\text { - The psychometric properties of the } \\
\text { instrument must be known. } \\
\text { - The instrument must not be diagnosis- } \\
\text { specific. }\end{array}$ & $\begin{array}{l}\text { - The instrument needs to be as short as possible } \\
\text { - There is as little overlap as possible with other } \\
\text { measurement instruments. }\end{array}$ \\
\hline \multicolumn{3}{|l|}{ Preferences } \\
\hline $\begin{array}{l}\text { - Preferably, the instrument is well-known and } \\
\text { already frequently used in practice. } \\
\text { - If possible, the instrument is frequently used in } \\
\text { an international setting. }\end{array}$ & $\begin{array}{l}\text { - The instrument is validated for Dutch } \\
\text { adults with } A B I \text {. }\end{array}$ & $\begin{array}{l}\text { - Preferably, all healthcare professionals should be } \\
\text { able to administer the instrument; no specific } \\
\text { training is required. } \\
\text { - The materials required for administration are as } \\
\text { few as possible. }\end{array}$ \\
\hline
\end{tabular}


proposed domain was important for the outcome measurement of persons with $\mathrm{ABI}$, with three response options ('yes', 'no' and 'this is not my area of expertise'). When this first question was answered affirmatively, the respondent was asked to indicate whether the measurement instruments proposed are suitable for the concerned domain ('yes', 'no' and 'no opinion'). The first round was aimed at identifying potentially suitable measurement instruments for the MDS-ABI. Accordingly, respondents were not asked to express a preference for a particular measurement instrument until the second round, but rather were asked to indicate whether a proposed instrument would be suitable for the MDS-ABI.

The aspects that healthcare interventions focus most on, such as reducing symptoms, minimising disability and improving quality of life, can only be assessed by patient-reported outcome measures. Moreover, patientreported outcome measures avoid observer bias and reduce the administrative burden of clinicians [28]. Therefore, respondents were stimulated to select patient-reported measurement instruments when appropriate. When respondents felt that a particular domain should be measured subjectively as well as objectively (by means of a test or observation measure), multiple instruments could be selected per domain in round one. Domains and instruments that reached a consensus of 'no' ( $\geq 51 \%$ of the respondents) were not taken into the next round.

Throughout all rounds, respondents were given the opportunity to elaborate on their decisions and to recommend additional or alternative domains and measurement instruments. Domains or instruments that were not in our list but proposed $\geq$ four times (i.e. $>10 \%$ of the sample) were included with the proposed domains and instruments in the next round. Measurement instruments that did not meet the requirements, (e.g. by not being freely available) were not presented in further rounds. In order to guide decision making on measurement instruments for the MDS-ABI, the requirements for measurement instruments and samples of the actual measures could be consulted using a hyperlink. Collective responses were fed back to the participants anonymously in the next round, using an information letter that was sent to the participants by e-mail. Participants who did not complete one of the three rounds were excluded from further participation.

The first Delphi round yielded consensus on the inclusion of all proposed outcome domains. Despite the fact that consequences of ABI can occur in all of these domains, measuring the full range of applicable ICF domains would be beyond the scope of a minimal dataset. Therefore, identification of the core domains for the MDS-ABI was carried through in round two. In order to narrow the selection of key domains, we sorted the domains that reached consensus in round one ascendingly by the percentage of 'Yes' answers, and asked respondents to reassess the proposed domains, keeping in mind that a minimal dataset needs to be compact and can be composed only of domains that are applicable to all adults with $\mathrm{ABI}$.

Regarding domains for which no consensus was reached on the level of measurement instrument, participants were asked to indicate their preferences, by putting the proposed instruments in order of preference (first place $=$ most preferred, last is least preferred). For some domains (such as emotional functioning), the multiple instruments that were regarded as suitable for the MDS-ABI by respondents in the first round measured different constructs (behaviour and depression/anxiety, respectively). In these cases, respondents had to indicate whether they wanted both, one or none of the instruments to be included in the MDS-ABI. Domains and instruments for which consensus was reached were entered into the concept MDS-ABI.

For some domains, no suitable instrument was identified by the expert panel. Therefore, the use of a screening question was reviewed by the respondents in the third round. Moreover, the third Delphi round contained questions to help clarify the last issues regarding overlap between selected instruments.

\section{Analyses}

The surveys were conducted using Qualtrics software [29]. All analyses were conducted using SPSS statistical software, version 24 [30]. Descriptive statistics were used for responder characteristics. Frequencies were calculated for ratings on multiple choice questions, excluding respondents who indicated they had no opinion on the particular subject. For ranking questions, mean ranks, standard deviation of the mean ranks, sum of ranks and the number of times the instrument was most preferred (first ranks) were calculated to indicate group preferences.

The level of agreement in order to reach consensus in Delphi procedures is not clearly defined [31]. The research team set this level a priori on the majority of respondents (51\%) in the current study for multiple choice questions. Consensus for rating questions was defined as an item that has the lowest mean rank, combined with the highest number of first ranks. Any discrepancies between these two outcomes meant that consensus was not reached.

\section{Results}

\section{Participants}

Initially, we identified 43 potential experts, of which 34 (79\%) agreed to participate. These participants identified another 20 potential experts. Of these newly identified potential experts, $14(70 \%)$ agreed to participate, leading to the inclusion of 48 experts in total. Forty-five of the included experts responded to the first round $(94 \%$ 
response rate). Forty of these 45 experts responded to round two (89\%). These 40 experts were invited to round three, to which all (100\%) responded (Fig. 1). Experts were employed in a diverse range of occupations and worked in several sectors of health care. Table 2 displays relevant participant characteristics.

\section{Round 1}

In the first round, all 18 proposed domains were selected with consensus rates ranging between $60.5-100 \%$. For an overview of all domains and corresponding result, see table in Additional file 1. Seven additional domains were mentioned $\geq$ four times by the expert panel: voice and speech functions $(n=4)$, behaviour $(n=4)$, financial situation $(n=5)$, social network $(n=6)$, religion $(n=4)$, education $(n=6)$ and personality $(n=7)$. Because behaviour is not a separate category in the ICF model, but is covered by several other domains, such as 'regulation of emotion' within 'emotional functioning' [5] and instruments assessing behavioural problems were already proposed under 'emotional functioning', the domain 'behaviour' was not entered into subsequent rounds. Furthermore, although personality has proven to be an important predictor of outcomes after ABI [32], classifying it requires comprehensive testing and therefore it is not within the scope of the MDS-ABI. The remaining five domains were presented to the respondents in round two. Last, $\geq$ four participants noticed the overlap between 'movement-related functions' and 'mobility'. Therefore, from this point, these domains were merged under 'mobility'.

On instrument level, 35 of the instruments proposed by the authors were considered suitable for measurement of the corresponding domains. See table in Additional file 2 for the ratings per instrument. Immediate consensus on measurement level was achieved for the domains pain and comorbidity. Also, for these domains, no additional measurement instruments that met the requirements were proposed by the respondents.

No immediate consensus was reached for the remaining domains. In addition, in the first round, the experts proposed three new instruments that complied with the requirements and were therefore advanced into the second round. Two of these instruments were intended for measuring energy/fatigue; the Dutch Multifactor Fatigue Scale [33] and the Checklist Individual Strength [34]. The third item that was proposed reflected the use of a screening question rather than a full measurement instrument for measuring social support: "Does the patient have an informal caregiver?"

Finally, within the domain 'injury characteristics', the definition of 'duration of hospital stay' and 'discharge destination' needed to be further clarified, judging by the

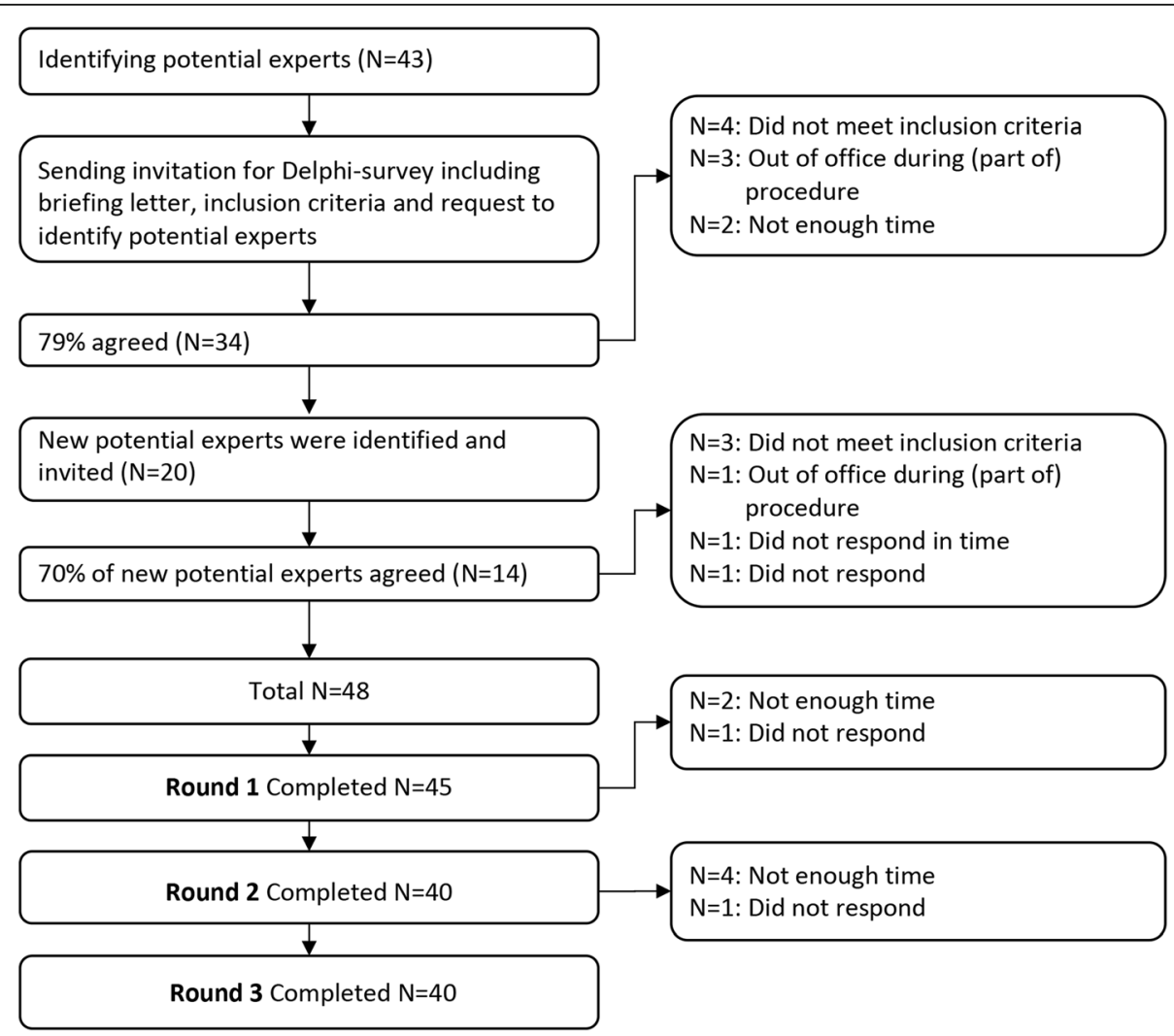

Fig. 1 Flow of participants in Delphi procedure 
Table 2 Characteristics of the expert panel $(n=45)$

\begin{tabular}{ll}
\hline Characteristic & Mean $\pm s d / n(\%)$ \\
\hline Sex: males, $n$ (\%) & $19(42)$ \\
Age in years, mean \pm (SD) & $48.1 \pm 11.5$ \\
Years of experience with developing/evaluating measurement instruments, mean $\pm(S D)$ & $16 \pm 11$ \\
Sector: $n$ (\%) & $7(9)$ \\
Elderly care & $14(18)$ \\
Rehabilitation & $9(12)$ \\
Mental health & $3(4)$ \\
Primary care & $5(6)$ \\
Disability care & $17(22)$ \\
Hospital & $18(23)$ \\
Higher education & $4(5)$ \\
Other & $18(40)$ \\
Occupation: $n$ (\%) & $12(27)$ \\
Psychologist & $11(24)$ \\
Physician & $4(9)$ \\
Researcher & \\
Other healthcare professionals & \\
\hline
\end{tabular}

${ }^{\mathrm{a}}$ Multiple entries were allowed

feedback of the respondents. Therefore, more detailed definitions ('duration of hospital stay after acquiring the brain injury' and 'discharge destination from hospital stay', respectively) were proposed to the respondents in round two.

\section{Round 2}

In the second round, consensus was reached on a total of 12 domains; these make up the domains of the concept MDS-ABI (Additional file 1). For all of the selected domains, consensus on measurement level was reached (Additional file 3).

For the domains 'communication' and 'support', the majority of the respondents opted for the use of a screening question, leading the research team to formulate and propose a screening question for the domain 'communication' to the respondents in round three. The screening question that had been proposed by one of the participants in round one was proposed to the panel in round two. However, the results pointed to the need for rephrasing the screening question, as it was deemed inappropriate by four participants. Therefore, in round three, we asked whether respondents would prefer the screening question "Do you experience sufficient support from the people in your surroundings?" over "Does the patient have an informal caregiver?".

Furthermore, there was some overlap between the instruments that were selected for mobility and self-care. The instrument that was selected to measure 'mobility' in the MDS-ABI, the Functional Ambulation Categories (FAC) [35], showed a clear resemblance to the instrument that was selected to measure 'self-care', the Barthel Index [36]. For instance, both instruments addressed the ability of ABI-patients to make transfers, to walk and to ascend stairs. Moreover, the satisfaction subscale of the Utrecht Scale for Evaluation of Participation - Rehabilitation (USER-P) [37] that was selected to measure 'participation', and the Life Satisfaction Questionnaire (LiSat) [38] that was favored to measure 'quality of life' showed significant overlap as well. Therefore, in the subsequent round, participants were asked to indicate which of these instruments should be selected for the concept MDS-ABI. Last, the majority of respondents agreed with the proposed definitions for 'duration of hospital stay' and 'discharge destination'. These newly defined variables were taken into the next round so that respondents could judge their suitability.

\section{Round 3}

On the question of how to settle the overlap between the measures that were selected for the domains 'mobility' and 'self-care', $83 \%$ of the respondents opted to drop the FAC and instead measure both domains with the Barthel Index. Also, both screening questions that were proposed to measure the 'communication' and 'social support' domains were agreed on by 90 and $82 \%$ of the respondents, respectively. Last, $62.5 \%$ opted for the inclusion of both newly defined variables 'duration of hospital stay' and 'discharge destination'. On the contrary, no consensus was reached on how to solve the overlap between measures of participation and quality of care. For more information about the content of the third 
Table 3 Domains and outcome measures that were selected by the Delphi expert panel

\begin{tabular}{|c|c|}
\hline ICF chapter & Selected instrument or variable \\
\hline \multicolumn{2}{|l|}{ Selected domain } \\
\hline \multicolumn{2}{|l|}{ Disease characteristics } \\
\hline Injury characteristics & $\begin{array}{l}\text { Date of brain injury, type of brain injury, previous brain injury, } \\
\text { duration of hospital stay, discharge destination. }\end{array}$ \\
\hline Comorbidity & CIRS \\
\hline \multicolumn{2}{|l|}{ Body functions \& structure } \\
\hline Cognitive functioning & MoCA \\
\hline Emotional functioning & HADS \\
\hline Energy & FSS \\
\hline \multicolumn{2}{|l|}{ Activities and participation } \\
\hline Mobility and Self-care & $\mathrm{Bl}$ \\
\hline Communication & $\begin{array}{l}\text { "Since acquiring brain injury, does the patient have trouble } \\
\text { communicating (expressing and/or understanding)?" }\end{array}$ \\
\hline Participation & USER-P \\
\hline Social support & $\begin{array}{l}\text { "Do you experience sufficient support from the people in } \\
\text { your surroundings?" }\end{array}$ \\
\hline \multicolumn{2}{|l|}{ Personal factors } \\
\hline Demographic characteristics & Age, sex, living situation, marital status, children. \\
\hline \multicolumn{2}{|l|}{ Other } \\
\hline Quality of life & No consensus \\
\hline
\end{tabular}

CIRS Cumulative Illness Rating Scale [39], MoCA Montreal Cognitive Assessment [40], HADS Hospital Anxiety and Despression Scale [41], FSS Fatigue Severity Scale [42], BI Barthel Index [36], USER-P Utrecht Scale for Evaluation of Rehabilitation - Participation [37]

survey, please refer to the table in Additional file 4. Table 3 displays an overview of the first concept version of the MDS-ABI, drafted after the results of the current Delphi study.

\section{Discussion}

The current study employed a Delphi procedure to define the content of a minimal dataset for outcome measurement in the field of ABI. The expert panel identified 12 domains of importance and reached consensus on how to measure 11 out of the 12 selected domains.

No consensus was reached on how to settle the overlap between the measurement instruments that were selected for the domains 'participation' and 'quality of life'. Respondents showed a clear pattern of reasoning for their decisions: experts either wanted to limit the length of the MDS-ABI and thus chose the USER-P, or considered it important to include a validated measure of quality of life, leading them to choose the USER-P in combination with the 12-Item Short Form Health Survey (SF-12) [43]. Since this dichotomy reflects a matter of personal preference, we believe a supplementary Delphi round would have yielded similar results. Initially, we adopted the inclusion of both the USER-P and the SF-12 in the MDS-ABI, considering the fact that this option received the highest preference scores. However, after the first draft version of the MDS-ABI was established, it appeared that the SF-12 - although freely available - cannot be scored without the use of purchased software. Consequently, the answer with the second-highest preference scores, i.e. applying the USER-P for measuring both 'participation' and 'quality of life', was put into practice in constructing the concept MDS-ABI. As a result, the MDS-ABI does not strictly cover a measure of quality of life.

\section{Features of the draft MDS-ABI}

Administration of the clinician-rated elements of the draft MDS-ABI will take approximately 25-40 min, based on the added average administration duration of all the measurement instruments. Completion time for the patient-reported questionnaires and screening questions is approximately $30 \mathrm{~min}$. Because of its wide array of outcome domains and relatively limited administration length, the MDS-ABI could be used for screening purposes in health care to assist anamnesis, diagnosis and determination of appropriate treatment and to serve as an outcome measure in research contexts. The draft MDS-ABI contains existing measures that are available in Dutch as well as other languages; most of these measures are frequently used in international settings. All measurement instruments are of sufficient methodological quality and are accessible without restrictions. The measurement instruments and screening questions 
are not specific to conditions within the spectrum of ABI. Therefore, the MDS-ABI can facilitate quick, standardised data collection on all ABI-patients.

Since the current MDS-ABI consists of several patientreported outcome measures, it is intended for use with adult patients who are capable of self-reporting on suitable domains. For ABI patients who are unable to complete these questionnaires, a so-called 'proxy-module' for all possible domains will be developed which can be administered by a professional or informal caregiver to retrieve essential information on injury outcomes. Likewise, since the MDS-ABI contains questionnaires that are irrelevant for patients in the acute phase of their injury, the authors recommend administering the HADS, FSS, and USER-P only in later stages of ABI.

Given that the current study was aimed at identifying important outcome domains for patients with ABI, no measurement instrument was included with regard to caregiver burden. Although ABI can induce a great and lasting burden on caregivers [44], identifying a suitable measure for the caregivers of persons with ABI was beyond the scope of this research. Future steps in the development of the MDS-ABI need to focus on incorporating caregiver experiences as well, especially in the proxy-module.

\section{Strengths and limitations}

The use of a Delphi procedure is a particular strength of the current study. As a Delphi procedure usually is undertaken through multiple rounds of surveys, it allows respondents to adjust their opinion based on other participants' knowledge, thereby transforming several personal opinions into a broad consensus [45]. Responses are anonymous; therefore, the Delphi procedure incorporates the advantages of group-based decision making without the disadvantages of face-to-face meetings such as practical implications and dominant characters [46].

The Delphi panel was composed of 45 healthcare professionals employed in a variety of sectors and disciplines of ABI health care. Accordingly, the results are considered to reflect the general opinion of experts across the field. In consulting experts rather than launching an established MDS-ABI, we anticipated stimulating bottom-up evolution of the dataset to improve support for the use of the MDS-ABI.

Given the fact that experts could access every proposed measurement instrument using survey hyperlinks, we estimate that respondents based their opinions on the quality and appropriateness of measurement instruments rather than familiarity alone.

The current study has several limitations. First, despite of the diversity of the healthcare sectors and disciplines represented by the Delphi panel, the sample was drawn from the professional network of the research team and consisted solely of Dutch participants; hence, it may not represent the opinion of all experts in the national and international field. Nonetheless, all selected measurement instruments are available in English and therefore, the MDS-ABI is suitable for international use. Nonwithstanding, we acknowledge that preferences for specific instruments may differ among countries. For instance, to date, the USER-P has mostly been used in Dutch studies.

Second, persons with ABI were not part of the actual Delphi procedure, although they defined crucial outcome domains during a group meeting preceding round one. ABI patients were not enrolled in the surveys because they lack expertise on measurement instruments and their psychometric properties. However, the experiences of persons with ABI will be evaluated in the consecutive feasibility study, in which the relevance of the selected domains and measures will be evaluated.

Moreover, some remarks should be made on the selected measurement instruments in the MDS-ABI. As the aim of a minimal dataset is to serve as a screening instrument of confined length, the selection of measurement instruments was limited to instruments with a short administration duration. A comprehensive assessment of certain domains arguably offers a better understanding of complaints than could be obtained by using shorter screening instruments. Similarly, there are widely used instruments of high quality that have been developed for patients with a specific diagnosis. Although the inclusion of such measures would not be feasible for a minimal dataset aimed at the heterogeneous group of people with $\mathrm{ABI}$, comprehensive measures such as personality questionnaires, and measures designed for specific diagnoses such as scales assessing arm functioning after stroke, can be added to the MDS-ABI to preference.

Furthermore, only those instruments that were validated and translated into Dutch at the time of the first round of the Delphi procedure complied with the requirements and were proposed to the participants. As a result, recently developed measures were not considered. As outcome measurement after $\mathrm{ABI}$ is a developing field, the MDS-ABI will need to be updated regularly in the future.

Last, no suitable instrument was identified for measuring the domains 'communication' and 'social support'. Since both domains were marked as essential for the MDS-ABI, respondents opted to use screening questions. As no validated screening questions for these domains exist, these items were composed by the Delphi panel. Therefore, their sensitivity and feasibility need to be evaluated in future research.

\section{Future research}

As a next step, we aim to evaluate the feasibility, usefulness and relevance of the MDS-ABI with healthcare 
professionals and with $A B I$ patients in several sectors of Dutch health care. On the long term, the MDS-ABI needs to be updated according to future developments in the field of minimal data collection, such as the development and implementation of comprehensive item banks. Such item bank systems, for example PROMIS [11], can be administered more efficiently using Computerized Adaptive Testing (CAT) [47]. However, this application is still under construction for most domains and therefore currently unavailable for use as part of the MDS-ABI. Once fully operative, CAT-administered item banks of the self-reported domains could be integrated into the MDS-ABI. Nonetheless, we value the additional clinician-rated parts of the MDS-ABI to obtain a complete image of the patient's status.

\section{Conclusions}

By performing a three-round Delphi study, the current study achieved consensus on the content of an ABIspecific minimal dataset. The expert panel selected twelve outcome domains (demographics, injury characteristics, comorbidity, cognitive functioning, emotional functioning, energy, mobility, self-care, communication, participation, social support and quality of life), measured with six existing measurement instruments (CIRS, MoCA, HADS, FSS, BI, USER-P), two screening questions, and registry of demographic and injury information. No consensus was reached on how to measure quality of life.

By developing an ABI-specific minimal dataset, we aim to facilitate uniform data collection to increase comparability, promote data pooling, and improve communication in the field of ABI. Furthermore, we hope to relieve the administrative burden for both patients and clinicians. The draft version of the MDS-ABI that is under development is based on the outcomes of the Delphi procedure and will soon be further evaluated and adjusted if necessary.

\section{Supplementary information}

Supplementary information accompanies this paper at https://doi.org/10 1186/s12955-020-01286-3.

Additional file 1. Results of voting for domains to be included in the MDS-ABI. Results are displayed on a dichotomous scale, excluding 'this is not my area of expertise'. Domains that that reached consensus in the second round were included in the draft MDS-NAH.

Additional file 2. Results of voting for suitable measurement instruments per domain in round 1. Results are displayed on a dichotomous scale, excluding respondents who indicated 'no opinion'.

Additional file 3. Responses (percentages) on questions about suitable measurement instruments per domain in round two. Table reflects domains for which multiple instruments measuring different constructs were selected in the previous round.

Additional file 4. Percentages of 'yes' on response categories of questions regarding the design of the concept MDS-ABI in round three.

\section{Abbreviations}

ABI: Acquired Brain Injury; BI: Barthel Index; CIRS: Cumulative Illness Rating Scale; FAIR: Findeable, Accesible, Interoperable and Reusable; FSS: Fatigue Severity Scale; HADS: Hospital Anxiety and Despression Scale;

ICF: International Classification of Functioning, Disability and Health; ICHOM: International Consortium for Health Outcomes Measures; LiSat: Life Satisfaction Questionnaire; MDS-ABI: Minimal Dataset for adults with ABI; MoCA: Montreal Cognitive Assessment; NIH: National Institute of Health; SF12: 12-Item Short Form Health Survey; TBI: Traumatic Brain Injury; USER-

P: Utrecht Scale for Evaluation of Rehabilitation - Participation

\section{Acknowledgements}

We would like to thank the Delphi panellists for their participation in this consensus study.

\section{Authors' contributions}

All of the authors contributed to the conceptualization, contributed content and participated in the development of the final manuscript. All authors read and approved the final manuscript.

\section{Funding}

Financial support for this work was received from the 'Gewoon Bijzonder' Programme on behalf of the Organisation of Health Research and Development (ZonMw - The Netherlands; www.zonmw.nl) (Grant reference 80-84500-98-113), cofinanced by the Brain Foundation of The Netherlands (Grant reference PZ 2016.02.1). The funders had no role in study design, data collection and analysis, decision to publish, or preparation of the manuscript.

\section{Availability of data and materials}

All data generated or analysed during this study are included in this published article and its supplementary information files.

\section{Ethics approval and consent to participate}

The current study did not need approval of the ethical board of the institution, as it did not regard patient information and did not influence patient care. Collected information was on expert opinions only. Regardless, potential experts were informed with the study objectives and procedures. Data was stored according to the Dutch personal data protection act. Participants provided informed consent by agreeing to the invitation that they received.

\section{Consent for publication}

Not applicable.

\section{Competing interests}

The authors declare that they have no competing interests.

\section{Author details}

${ }^{1}$ School for Mental Health and Neuroscience, Faculty of Health, Medicine and Life Sciences, Maastricht University Medical Center, Maastricht, The Netherlands. ${ }^{2}$ Limburg Brain Injury Center, Maastricht, The Netherlands. ${ }^{3}$ Department of Neuropsychology and Psychopharmacology, Faculty of Psychology and Neuroscience, Maastricht University, Maastricht, The Netherlands. ${ }^{4}$ Care and Public Health Research Institute, Faculty of Health, Medicine and Life Sciences, Maastricht University Medical Center, Maastricht, The Netherlands. ${ }^{5}$ Department of Research and Development, Heliomare Rehabilitation Center, Wijk aan Zee, The Netherlands. ${ }^{6}$ Coronel Institute of Occupational Health, Amsterdam Public Health Research Institute, Academic Medical Center, University of Amsterdam, Amsterdam, The Netherlands.

Received: 26 June 2018 Accepted: 10 February 2020

Published online: 17 February 2020

\section{References}

1. Pereira S, Richardson M, Mehta S, Teasell R, Miller T. Toning it down: selecting outcome measures for spasticity management using a modified Delphi approach. Arch Phys Med Rehabil. 2015:96(3):518-23.e18.

2. Tate RL, Godbee K, Sigmundsdottir L. A systematic review of assessment tools for adults used in traumatic brain injury research and their relationship to the ICF. NeuroRehabilitation. 2013;32(4):729-50. 
3. Polinder S, Haagsma JA, van Klaveren D, Steyerberg EW, van Beeck EF. Health-related quality of life after TBI: a systematic review of study design, instruments, measurement properties, and outcome. Popul Health Metrics. 2015;13:4.

4. Tse T, Douglas J, Lentin P, Carey L. Measuring participation after stroke: a review of frequently used tools. Arch Phys Med Rehabil. 2013:94(1):177-92.

5. World Health Organization. International Classification of Functioning, Disability and Health: ICF. Geneva: World Health Organization; 2001.

6. Wilkinson MD, Dumontier M, Aalbersberg IJ, Appleton G, Axton M, Baak A, et al. The FAIR guiding principles for scientific data management and stewardship. Sci Data. 2016;3:160018.

7. Haywood KL, Griffin XL, Achten J, Costa ML. Developing a core outcome set for hip fracture trials. Bone Joint J. 2014;96-B(8):1016-23.

8. Koke AJ, Smeets RJ, Schreurs KM, van Baalen B, de Haan P, Remerie SC, et al. Dutch dataset pain rehabilitation in daily practice: content, patient characteristics and reference data. Eur J Pain. 2017;21(3):434-44

9. Lutomski JE, Baars MA, Schalk BW, Boter H, Buurman BM, den Elzen WP, et al. The development of the older persons and informal caregivers survey minimum DataSet (TOPICS-MDS): a large-scale data sharing initiative. PLoS One. 2013;8(12):e81673

10. Hicks R, Giacino J, Harrison-Felix C, Manley G, Valadka A, Wilde EA. Progress in developing common data elements for traumatic brain injury research: version two--the end of the beginning. J Neurotrauma. 2013:30(22):1852-61.

11. Cella D, Riley W, Stone A, Rothrock N, Reeve B, Yount S, et al. The patientreported outcomes measurement information system (PROMIS) developed and tested its first wave of adult self-reported health outcome item banks: 2005-2008. J Clin Epidemiol. 2010;63(11):1179-94.

12. Gershon RC, Lai JS, Bode R, Choi S, Moy C, Bleck T, et al. Neuro-QOL: quality of life item banks for adults with neurological disorders: item development and calibrations based upon clinical and general population testing. Qual Life Res. 2012;21(3):475-86

13. Gershon RC, Cella D, Fox NA, Havlik RJ, Hendrie HC, Wagster MV. Assessment of neurological and behavioural function: the NIH toolbox. Lancet Neurol. 2010;9(2):138-9.

14. Kelley TA. International consortium for health outcomes measurement (ICHOM). Trials. 2015;16(3):O4

15. Salinas J, Sprinkhuizen SM, Ackerson T, Bernhardt J, Davie C, George MG, et al. An international standard set of patient-centered outcome measures after stroke. Stroke. 2016;47(1):180-6.

16. Williamson PR, Altman DG, Blazeby JM, Clarke M, Devane D, Gargon E, et al. Developing core outcome sets for clinical trials: issues to consider. Trials. 2012;13(1):132.

17. Dalkey N, Helmer O. An experimental application of the Delphi method to the use of experts. Manag Sci. 1963;9(3):458-67.

18. Boulkedid R, Abdoul H, Loustau M, Sibony O, Alberti C. Using and reporting the Delphi method for selecting healthcare quality indicators: a systematic review. PLoS One. 2011;6(6):e20476.

19. Geyh S, Cieza A, Schouten J, Dickson H, Frommelt P, Omar Z, et al. ICF Core sets for stroke. J Rehabil Med. 2004;36(0):135-41.

20. Bernabeu M, Laxe S, Lopez R, Stucki G, Ward A, Barnes M, et al. Developing core sets for persons with traumatic brain injury based on the international classification of functioning, disability, and health. Neurorehabil Neural Repair. 2009:23(5):464-7.

21. McDougall J, Wright $V$, Rosenbaum $P$. The ICF model of functioning and disability: incorporating quality of life and human development. Dev Neurorehabil. 2010;13(3):204-11.

22. Whiteneck G, Dijkers MP. Difficult to measure constructs: conceptual and methodological issues concerning participation and environmental factors. Arch Phys Med Rehabil. 2009:90(11):S22-35.

23. Maas Al, Menon DK, Steyerberg EW, Citerio G, Lecky F, Manley GT, et al. Collaborative European NeuroTrauma effectiveness research in traumatic brain injury (CENTER-TBI) a prospective longitudinal observational study. Neurosurgery. 2014;76(1):67-80.

24. Mierlo ML, Heugten CM, Post MW, Lindeman E, Kort PL, Visser-Meily J. A longitudinal cohort study on quality of life in stroke patients and their partners: Restore4Stroke cohort. Int J Stroke. 2014:9(1):148-54.

25. van Heugten CM, Ponds R, Berkers R, Smeets S, Gregório-Claessens GW. Zorgmonitor NAH in de GGZ: een onderzoek naar de zorgbehoeftes en zorgverlening voor klinische patiënten met niet aangeboren hersenletsel in de GGZ; 2012
26. Huijsman R. CVA Ketenzorg 3.0. Innoveren in houdbare zorg is topsport. Berlin: Springer; 2013. p. 86-9.

27. Tate RL. A compendium of tests, scales, and questionnaires: the practitioner's guide to measuring outcomes after acquired brain impairment. London: Psychology Press; 2010.

28. Black N. Patient reported outcome measures could help transform healthcare BMJ. 2013:346:1167.

29. Qualtrics L. Qualtrics [software]. Utah: Qualtrics; 2014

30. IBM Corporations. IBM SPSS statistics for Macbook (version 24.0). Armonk: IBM Corporations; 2016.

31. von der Gracht HA. Consensus measurement in Delphi studies. Technol Forecast Soc Chang. 2012;79(8):1525-36.

32. van Mierlo M, van Heugten C, Post MW, Hoekstra T, Visser-Meily A. Trajectories of health-related quality of life after stroke: results from a oneyear prospective cohort study. Disabil Rehabil. 2017:40:1-10.

33. Visser-Keizer AC, Hogenkamp A, Westerhof-Evers HJ, Egberink IJ, Spikman JM. Dutch multifactor fatigue scale: a new scale to measure the different aspects of fatigue after acquired brain injury. Arch Phys Med Rehabil. 2015; 96(6):1056-63.

34. Vercoulen JH, Swanink CM, Fennis JF, Galama JM, van der Meer JW, Bleijenberg G. Dimensional assessment of chronic fatigue syndrome. J Psychosom Res. 1994:38(5):383-92.

35. Holden MK, Gill KM, Magliozzi MR, Nathan J, Piehl-Baker L. Clinical gait assessment in the neurologically impaired: reliability and meaningfulness. Phys Ther. 1984;64(1):35-40.

36. Mahoney Fl, Barthel DW. Functional evaluation: the Barthel Index: a simple index of independence useful in scoring improvement in the rehabilitation of the chronically ill. Md State Med J. 1965;14:61-5.

37. Post MW, van der Zee CH, Hennink J, Schafrat CG, Visser-Meily JM, van Berlekom SB. Validity of the Utrecht scale for evaluation of rehabilitationparticipation. Disabil Rehabil. 2012;34(6):478-85.

38. Fugl-Meyer AR, Bränholm I-B, Fugl-Meyer KS. Happiness and domain-specific life satisfaction in adult northern swedes. Clin Rehabil. 1991;5(1):25-33.

39. Linn BS, Linn MW, Gurel L. Cumulative illness rating scale. J Am Geriatr Soc. 1968:16(5):622-6.

40. Nasreddine ZS, Phillips NA, Bédirian V, Charbonneau S, Whitehead V, Collin I, et al. The Montreal cognitive assessment, MoCA: a brief screening tool for mild cognitive impairment. J Am Geriatr Soc. 2005;53(4):695-9.

41. Zigmond AS, Snaith RP. The hospital anxiety and depression scale. Acta Psychiatr Scand. 1983;67(6):361-70.

42. Krupp LB, LaRocca NG, Muir-Nash J, Steinberg AD. The fatigue severity scale: application to patients with multiple sclerosis and systemic lupus erythematosus. Arch Neurol. 1989;46(10):1121-3

43. Ware JE Jr, Kosinski M, Keller SD. A 12-item short-form health survey: construction of scales and preliminary tests of reliability and validity. Med Care. 1996:34(3):220-33.

44. Harding R, Gao W, Jackson D, Pearson C, Murray J, Higginson IJ. Comparative analysis of informal caregiver burden in advanced cancer, dementia, and acquired brain injury. J Pain Symptom Manag. 2015;50(4): 445-52.

45. Hasson F, Keeney S, McKenna H. Research guidelines for the Delphi survey technique. J Adv Nurs. 2000;32(4):1008-15.

46. Murry JW, Hammons JO. Delphi: a versatile methodology for conducting qualitative research. Rev High Educ. 1995;18(4):423-36.

47. Fries JF, Witter J, Rose M, Cella D, Khanna D, Morgan-DeWitt E. Item response theory, computerized adaptive testing, and PROMIS: assessment of physical function. J Rheumatol. 2014:41(1):153-8.

\section{Publisher's Note}

Springer Nature remains neutral with regard to jurisdictional claims in published maps and institutional affiliations. 